\title{
Control System Design for Electric-Intensive Mobile Rack
}

\author{
Yang Haigen, Zhan Zhihuan, Fu Xiao and Wang Yong \\ Engineering Research Center of Wider and Wireless Communication \\ Technology, Ministry of Education, Nanjing University of Posts and \\ Telecommunications, China; \\ Beijing Institute of Specialized Machinery, Beijing, China \\ \{yhg,wangy,fuxiao\}@njupt.edu.cn; ailsa3484@163.com
}

\begin{abstract}
The land and space resources are very tight in this world today, in order to save storage space and improve the efficiency of the enterprise, this paper designs an electric-intensive mobile rack system. This system uses MCU as the controller, integrates multiple security protection system to improve the safety of the system, while the comprehensive control software ensures the stability of the system. The practice shows that this system can greatly reduce storage space and improve the efficiency of cargo access.
\end{abstract}

Keywords: mobile rack; $M C U$; control system; Rs-485; safety detection

\section{Introduction}

With the rapid development of the world economy and the progress of modern science and technology, the logistics industry as a service sector in the emerging economy is growing rapidly in worldwide. Warehouse management as the central part of the logistics is highly valued in the United States, Germany, Japan and other developed countries as early as the 1960s and 1970s. Automated warehouse technology as an important production based on Computer Integrated Manufacturing System (CIMS) technology had developed more mature in the late 1980s [1]. Because of better utilization of space the automated warehouse is more and more widely used. The fixed rack, automated warehouse has many advantages, but the biggest drawback is a waste of space. Many rack manufacturers design a type of mobile rack system. Mobile rack is a type of rack with wheels mounted on the bottom of the rack, which can be divided into two types according to the rack whether running on rail track or on non-rail. The most advantage of mobile rack is to save space and improve efficiency.

Goetschalckx Marc and McGinnis Leon in 2004 present several models for the design of warehousing systems that use modular drawers and bin shelving, storage technologies [2]; Wang, P G, Qi X M, Zong X P, et al., researched and analyzed the order-picking task of the fixed rack to improve the efficiency of automated warehouse [3]; Guezzen, Amine Hakim, Sari Zaki, Ghomri Latefa in 2011 given a system which consists of storage racks moving laterally on rails so that one can open an aisle between any two joint racks, storage/retrieval (S/R) machines, the control system and an input/output (I/O) station; Sari Z, Hamzaoui A in 2013 aim to determine the optimal dimensions of a flow rack automated storage and retrieval system (AS/RS) using a single machine for storage and retrieval operations [4]. These optimal dimensions are evaluated for minimum expected travel times of the storage and retrieval machine. Enumeration technique is used on a large range of system configurations going from 100 to 20000 storage segments in the rack. 
Most controllers of mobile rack are based on PLC. PLC has many advantages, but its price is so expensive. We proposed a Micro Control Unit (MCU) based mobile rack control system. This paper describes the hardware design and implementation of the controller and control software of the electric-intensive mobile rack system. The system has been running smoothly in a pharmaceutical company for 2 years.

The paper is organized as follows: Second II outlines the architecture and the main component of the electric-intensive mobile rack system; in section III shows the hardware design of the mobile rack; Section IV presents the design of the control software for the mobile rack and its application; The conclusions are brought out in Section V.

\section{Electric-Intensive Mobile Rack Systems Introduction}

\subsection{Mobile Rack System Architecture}

The electric-intensive mobile rack designed in this paper is a next generation storage device based on the development of traditional mobile rack. The system is running on the rail track laid on the ground by the chassis with wheels, which lateral move along the rail track driven by power and mechanical devices fixed on the chassis. When no storage jobs, there is no channel between the rack apart, tightly packed; When need to access productions, by remote control, make the racks to move some space in front of the appropriate rack to make the space as the channel of forklifts and other equipment. Obviously, this rack has the characteristics, such as high degree of automation, big storage capacity, small footprint, saving human resources and improves warehouse space utilization; improve work efficiency [5]. Figure 1 shows the architecture of the mobile rack. 


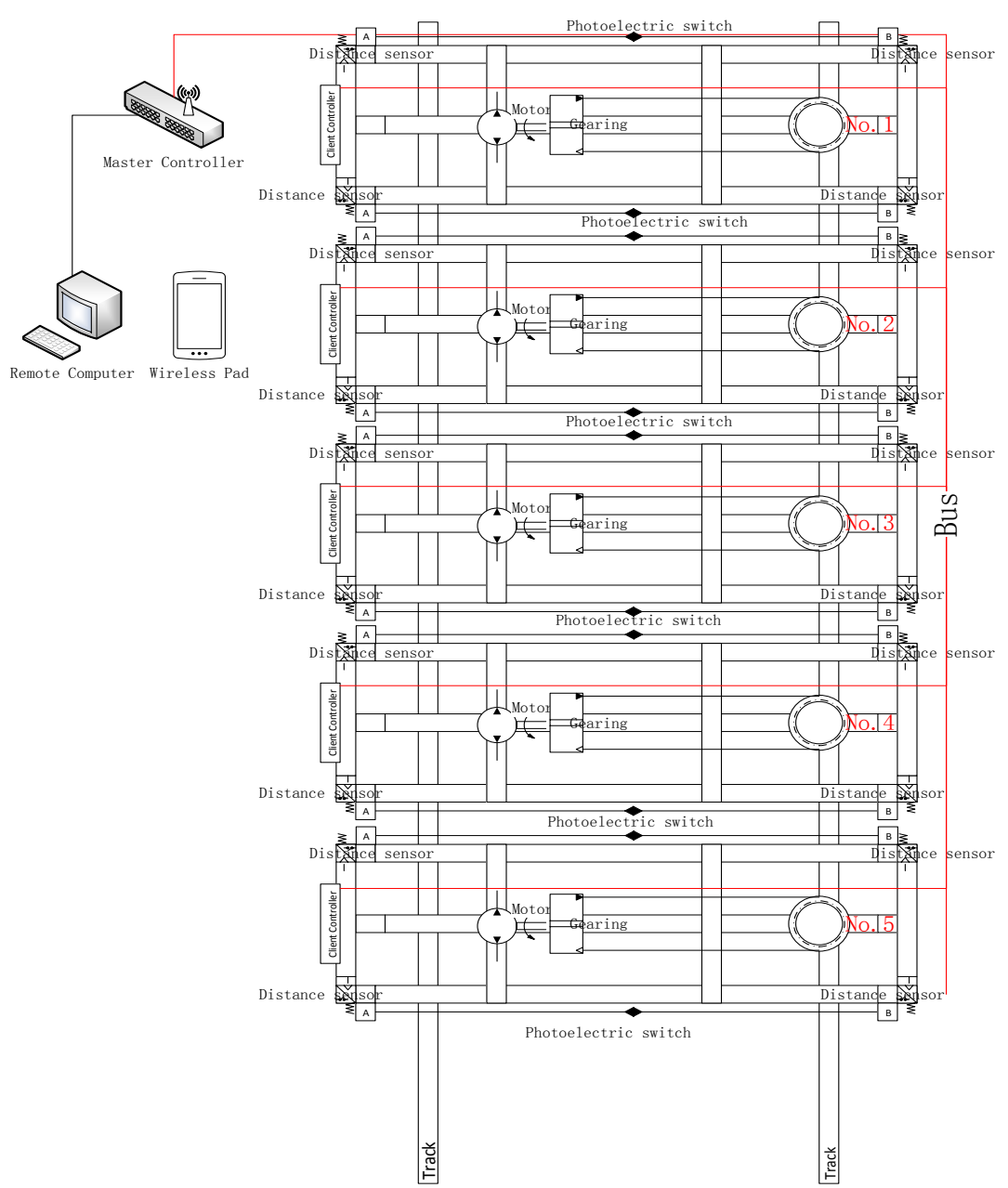

Figure 1. Architecture of the Mobile Rack

\subsection{The Main Parts of the Mobile Rack}

Here are the main parts of the mobile rack:

- Master Controller: Send the control commands to control the corresponding rack moves, thus out of the operating channel. Also the master controller communicates with remote computers or wireless pad via wired or wireless way to control the rack.

- Client Controller: Mounted on the rack for the movement control unit, control the execution unit on the rack to do move operation, while acquisition signals from various sensors on the rack, to achieve security detection and control.

- Bus: RS-485 bus, which is used to transmit control signals, the farthest can transfer $1.2 \mathrm{~km}$.

- Motor: Motor-driven mobile rack device. A three-phase alternating current motor, drive the transmission mechanism for driving to move the rack.

- Distance Sensor: Ultrasonic distance sensor. For detecting the distance between the two racks, thereby controlling the speed of movement of the rack.

- Photoelectric switch: When the rack is running somebody or something in collision with the rack, the photoelectric switch can detect the collision signal, the rack will stop running.

- Remote Computer\& Wireless Pad: The two devices send the control command by wired or wireless way to master controller to control the rack.

- Track: There are four wheels fixed on the bottom of the rack, the wheels running on the track. The difference between the rack with track and non-track is the rack 
with track can constrain movement of the rack, so as to move according to a predetermined route.

\section{The Controller Design for the Mobile Rack}

The architecture of the control system of the mobile rack is shown in Figure 2. The control system can be divided into two parts: master controller and client controller. The master controller consists of five main modules: button input module, led display module, Ethernet communication module, wireless communication module and Rs-485 communication module. The client controller consists of six modules: button input module, indicator light module, Rs-485 communication module, distance sensor input module, photoelectric switch input module and driven motor module.

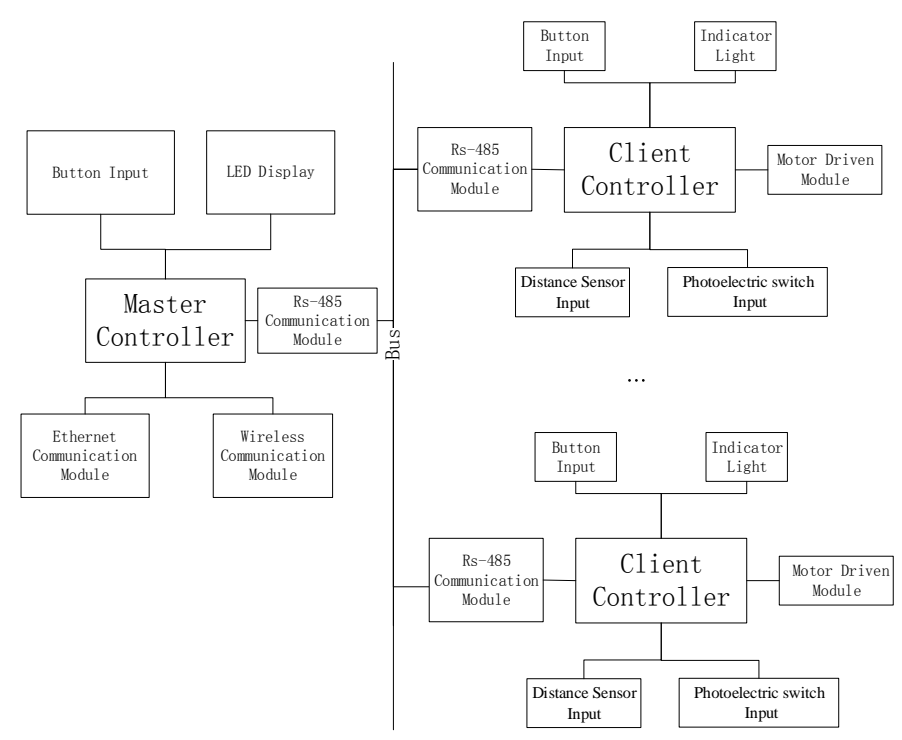

Figure 2. Architecture of the Control System

\subsection{The MCU System for the Controller}

In this paper, the core of the control circuit of the master or client controller is MCU system. The MCU is responsible for handling the response of button commands, input signals from various sensors, motor drives, 485/Ethernet/wireless communications, and display output. We use the STC's STC89c52RC microcontroller as the control chip. This MCU is fully compatible with 8051 , which working frequency is $48 \mathrm{MHz}$, program space is $8 \mathrm{~K}$ bytes, integrated $512 \times 8$-Bit internal RAM, 32 programmable I/O lines, three 16-bit timers, four Interrupt Sources.

In order to make the MCU work, the system of MCU must include: a quartz crystal oscillator; RESET Input; 5 Volt DC supply. The schematic diagram of the minimum system of MCU is shown in Figure 3. 


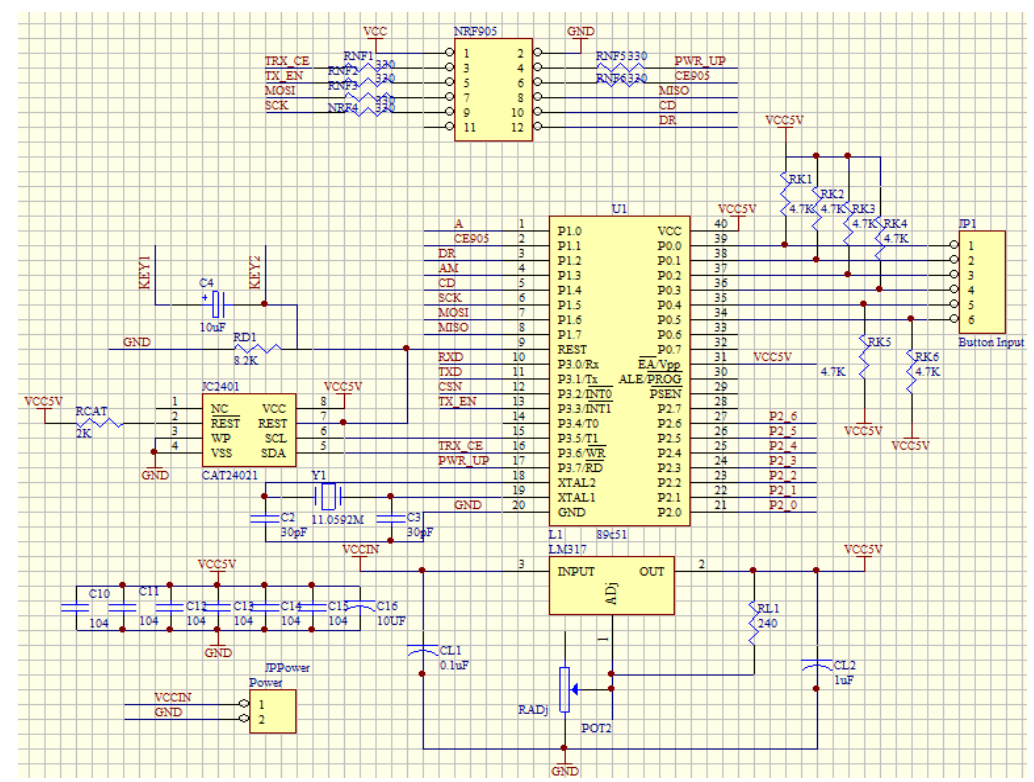

Figure 3. Schematic Diagram of MCU

- A quartz crystal oscillator (11.0592MHz) is connected to inputs XTAL1 (pin19) and XTAL2 (pin18) and also needs two capacitors of $30 \mathrm{pF}$.

- RESET input (Pin 9) pin is active high (normally low), upon applying a high pulse to this pin, the MCU will reset and terminate all activities. The system use CAT24021 do reset and watchdog.

- 5 Volt DC supply, The LM317 of adjustable 3-terminal positive voltage regulators can supply in excess of $1.5 \mathrm{~A}$ over a $1.2 \mathrm{~V}$ to $37 \mathrm{~V}$ output range. The output voltage is selected using two resistors. Normally RL1 is $240 \Omega$. The formula for calculating the value of $\mathrm{RADj}$ is Vout=1.25 (1+(RADj/RL1)), so the $\mathrm{RADj}$ is $720 \Omega$.

\subsection{The Communication Module}

The communication of the mobile rack includes: Rs-485 communication module, Ethernet communication module, wireless communication module. These modules are used to transfer the control commands and the sensor signal data.

- Rs-485 communication module

Rs-485 has high transmission speed, long transmission distance (as long as 1200m) and strong anti-clutter ability and many other advantages, and it allowed one sender unit to drive the many load equipment in one pair of twisted pair lines. In this paper, we use the Rs- 485 bus to achieve the transmission commands or signal data between the master and client controllers and used the MAX485 driving chip to carry on the level transformation. The schematic diagram of Rs-485 is shown in Figure 4.

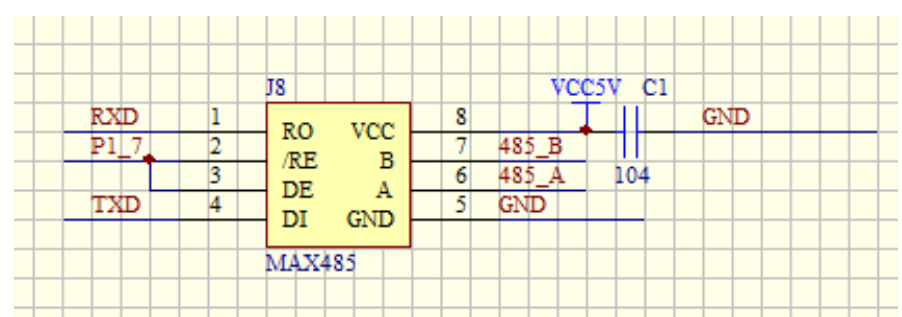

Figure 5. Schematic Diagram of Rs-485 
- Ethernet Communication Module

In order to realize the remote computer to control the mobile rack, this needs an Ethernet communication module to achieve the communication between the remote computer and the master controller. To save development time, we directly use the serial-to-Ethernet module of ZLG, which is shown in Figure 5.

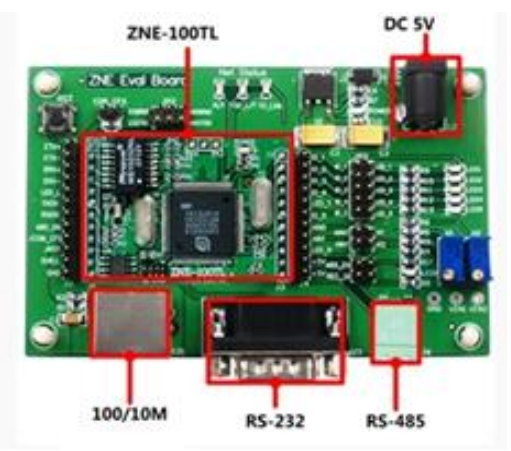

\section{Figure 6. Serial-to-Ethernet Module}

- Wireless Communication Module

There are many wireless transceiver modules on the market, such as Bluetooth wireless transceiver modules, nRF chips, et al. In this paper, we use nRF905 of Nordic as the wireless transceiver to transmit the data. The nRF905 radio works in $915 \mathrm{MHz}$ Industrial, Scientific and Medical (ISM) band and supports a bit rate of $100 \mathrm{kbps}$ with Manchester encoding, providing a data-link operating at $50 \mathrm{kbps}$. The nRF905 radio uses GFSK modulation and provides a transmission range of up to $1 \mathrm{~km}$ using standard unity-gain quarter-wavelength antennas. Figure 6 gives the nRF905 transceiver for communication.

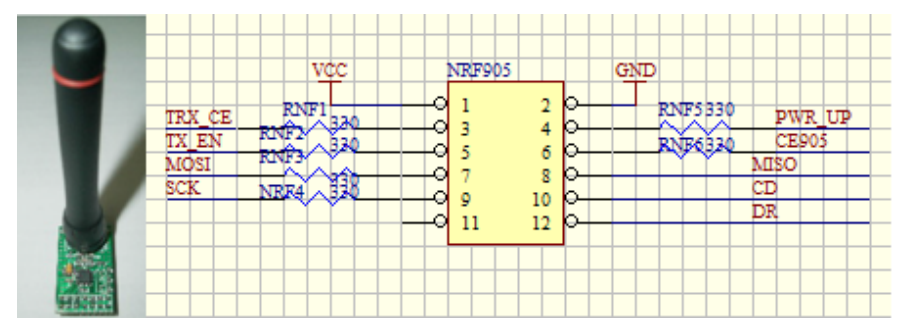

Figure 7. nRF905 Wireless Communication Module

\subsection{Motor Driven Module}

The mobile rack usually uses the DC or AC motor to drive to the rack, Satya Sheel and Omhari Gupta [6] in 2012 presented the design of fuzzy logic based PID controller for the overall nonlinear control system for the DC motor drive. In this paper uses three-phase AC motor to drive the rack. The MCU sends the signals to the solid state relays used to change their state from non-conducting to conducting, which can make the motor forward or reverse working. The Figure 7 shows the hardware of the motor driven system. 


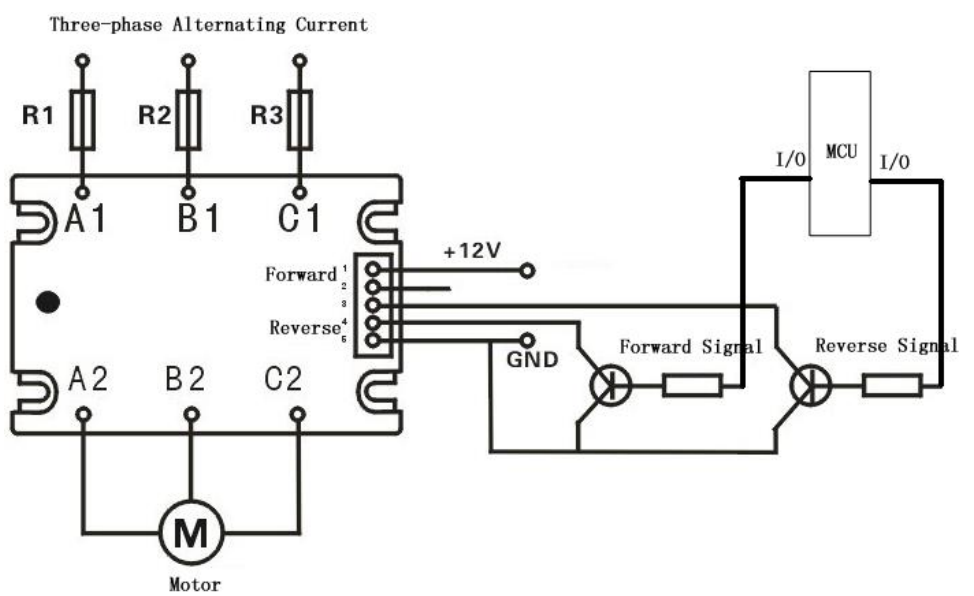

Figure 8. The Hardware of the Motor Driven System

In order to select the appropriate motor, needs to calculate the RPM of the motor.

(1) Estimate Motion of friction

$$
F_{1}=\frac{g(Q+C)(2 f+u d)}{D}
$$

where, $\mathrm{g}$ is the gravity, $9.8 \mathrm{~m} / \mathrm{s}^{2}$; Q is cargo deadweight; $\mathrm{C}$ is the weight of the rack; $\mathrm{f}$ is the friction coefficient of the wheel, which is $0.5 ; \mathrm{u}$ is the friction coefficient of the bearing, which is 0.004 ; $\mathrm{d}$ is the radius of the bearing; $\mathrm{D}$ is the external diameter of the wheel.

(2) Estimate the accelerating force

$$
F_{2}=(Q+C) a=0.05(Q+C)
$$

where, max of a is $0.05 \mathrm{~m}^{\mathrm{m}} / \mathrm{s}^{2}$,

(3) Estimate the resistance force due to the roughness of the track

$$
F_{3}=g(Q+C) \sin \alpha=0.003 g(Q+C)
$$

where, $\sin \alpha=0.003$ is the deviation due to the roughness of the track.

The total resistance is

$$
F=F_{1}+F_{2}+F_{3}
$$

(4) The power of the motor

$$
N \geq \frac{F V}{12}(K W)
$$

where $\mathrm{V}$ is less than $5 \mathrm{~m} / \mathrm{min}$

(5) Reduction ratio

$$
i=\frac{n_{1}}{n_{2}}
$$


where, $n_{1}$ is the rated speed of the motor; $n_{2}$ is wheel Speed.

$$
n_{2}=\frac{500}{\pi D}
$$

The actual selection of gear motors, according to motor power $\mathrm{N}$ and wheel speed $n_{2}$.

\subsection{The Safety Detection Device}

In order to ensure the safe operation of the rack, we use two types of protection measures: one is distance sensor, the second is photoelectric switch. As shown in Figure 8 , the distance sensor usually detects the distance between two racks to control the speed of the rack. When the rack is running, if anything blocking the car forward, the photoelectric switch can detect the collision signal, the rack will stop running.

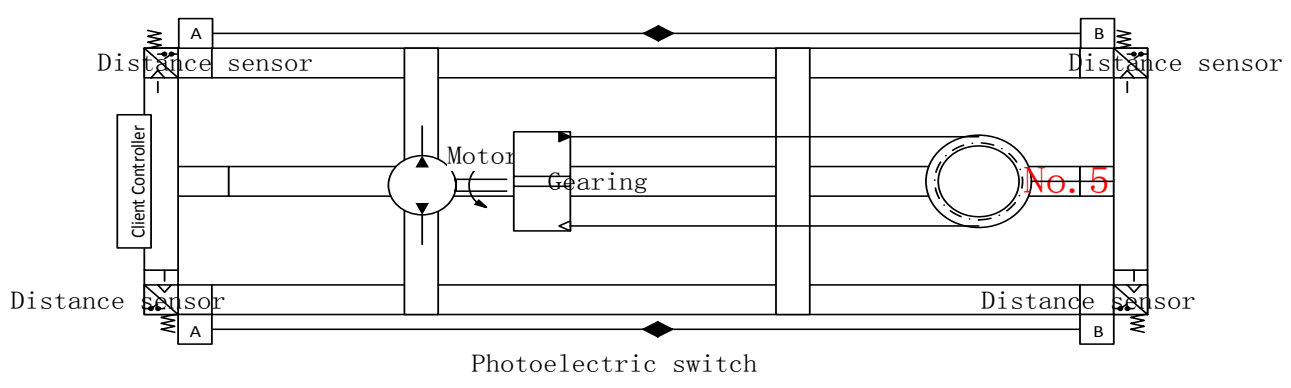

Figure 9. Safety Detection Device

\subsubsection{The Hardware of Distance Detection}

Because of the strong point of the ultrasonic energy consumption slow propagation in the medium distance and thus often used for ultrasonic distance measurement. So we use the ultrasonic ranging system to detect the distance between two racks.

1) Ultrasonic transmitter circuit

The ultrasonic transmitter circuit is mainly composed of an inverter 74LS04 and ultrasonic transducers, the MCU P1.0 port output a $40 \mathrm{kHz}$ square wave signal, one way through one stage of inverter transmit to an electrode of the ultrasonic transducer, another way through two stages of inverter transmit to another electrode of the ultrasonic transducer. By using the push-pull form method to add the square wave signal at both ends of the ultrasonic transducer can be improved the emission intensity of the ultrasonic. The schematic of the ultrasonic transmitter circuit is given in Figure 9.

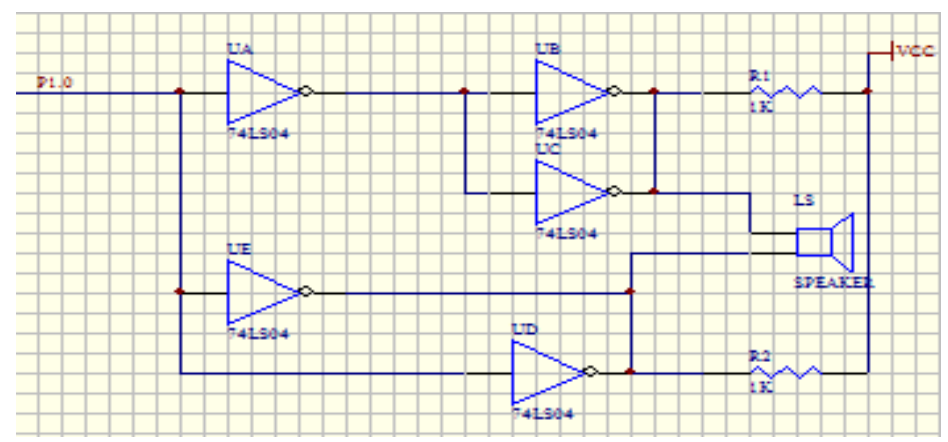

Figure 10. Schematics of the Ultrasonic Transmitter Circuit 
2) Ultrasonic detector receiver circuit

CX20106A is an infrared detector receiver chip, which usually used as TV infrared remote control receiver. Considering the CX20106A carrier frequency is $38 \mathrm{kHz}$ closer to the ultrasonic ranging frequency $40 \mathrm{kHz}$, we can use it to design an ultrasonic receiver detection circuit. Experiments show that, by using CX20106A to receive ultrasound with high sensitivity and strong anti-jamming capability. Figure 10 gives the schematics of the ultrasonic transmitter circuit.

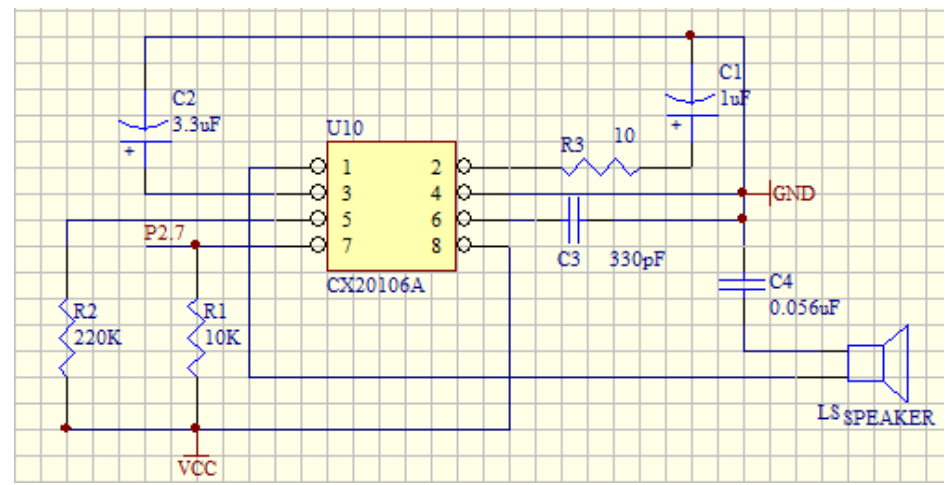

Figure 11. Schematics of the Ultrasonic Detector Receiver Circuit

\subsubsection{The Hardware of Photoelectric Switch}

When the rack is running somebody or something in collision with the rack, the photoelectric switch can detect the collision signal, the rack will stop running. In Figure 8 , we can see the A and B are two photoelectric switches, A is a light transmitter, B is a light receiver. If $\mathrm{B}$ cannot receive the light, this device will send a signal to the MCU. The MCU will make the rack stop running.

\section{The Control Software Design for the Mobile Rack}

In the above section, we described the hardware design of the control system for the electric-intensive mobile rack in detail. This section we will show the control algorithm and software design for the mobile rack.

Generally, the master control usually only deal with the button operation and command communication, no complex functions. In this section, we focus on the software design and the control algorithm of the client controller. Figure 11 shows the flowchart of the mobile rack. 


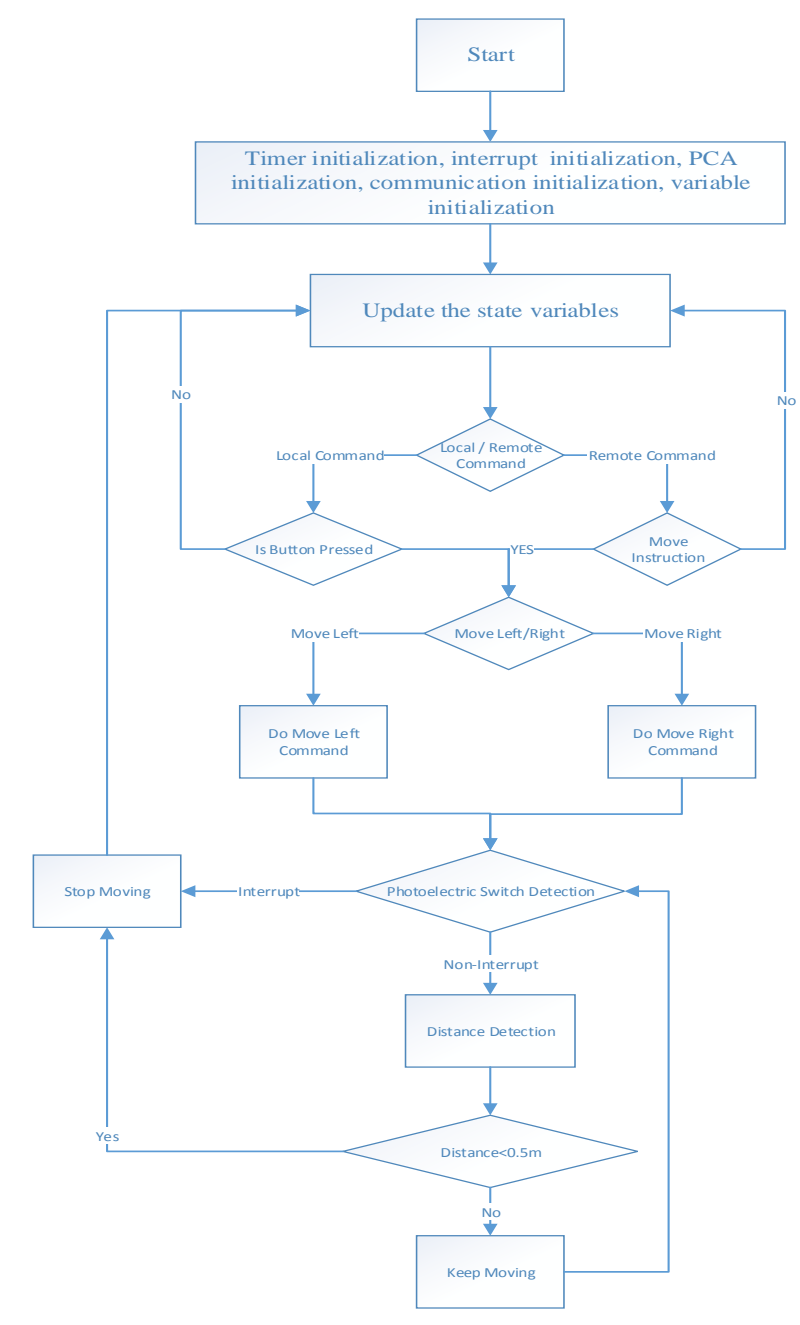

Figure 12. The Flow Chart of the Software of the Mobile Rack

\subsection{Master-Client Communication Based on 485 Bus}

As mentioned above, Rs-485 is a typical industrial field bus, which used in the communication between the master and client controllers. The system uses half-duplex master-slave mode to achieve communication.

A full communication process includes 3 stages: (1) the master query stage; (2) the slave reply stage; (3) link release stage. In the query stage, the master sends the address code, command, data and check code to the slave. In the reply stage, the slave will receive the command and data, check the check code and reply the information to the master. In the link release stage, the slave and the master will reset the buffer and variables, prepare for the next communication. This communication process has always started by the master, the slave is on listening. When the slave receives the two bytes of the address code, will check whether the address is its own address. If it is true, then continue to receive the rest of the data, else do nothing. When the slave is on listening and the master is on the Rs-485 communication workflow is shown in Figure 12. 


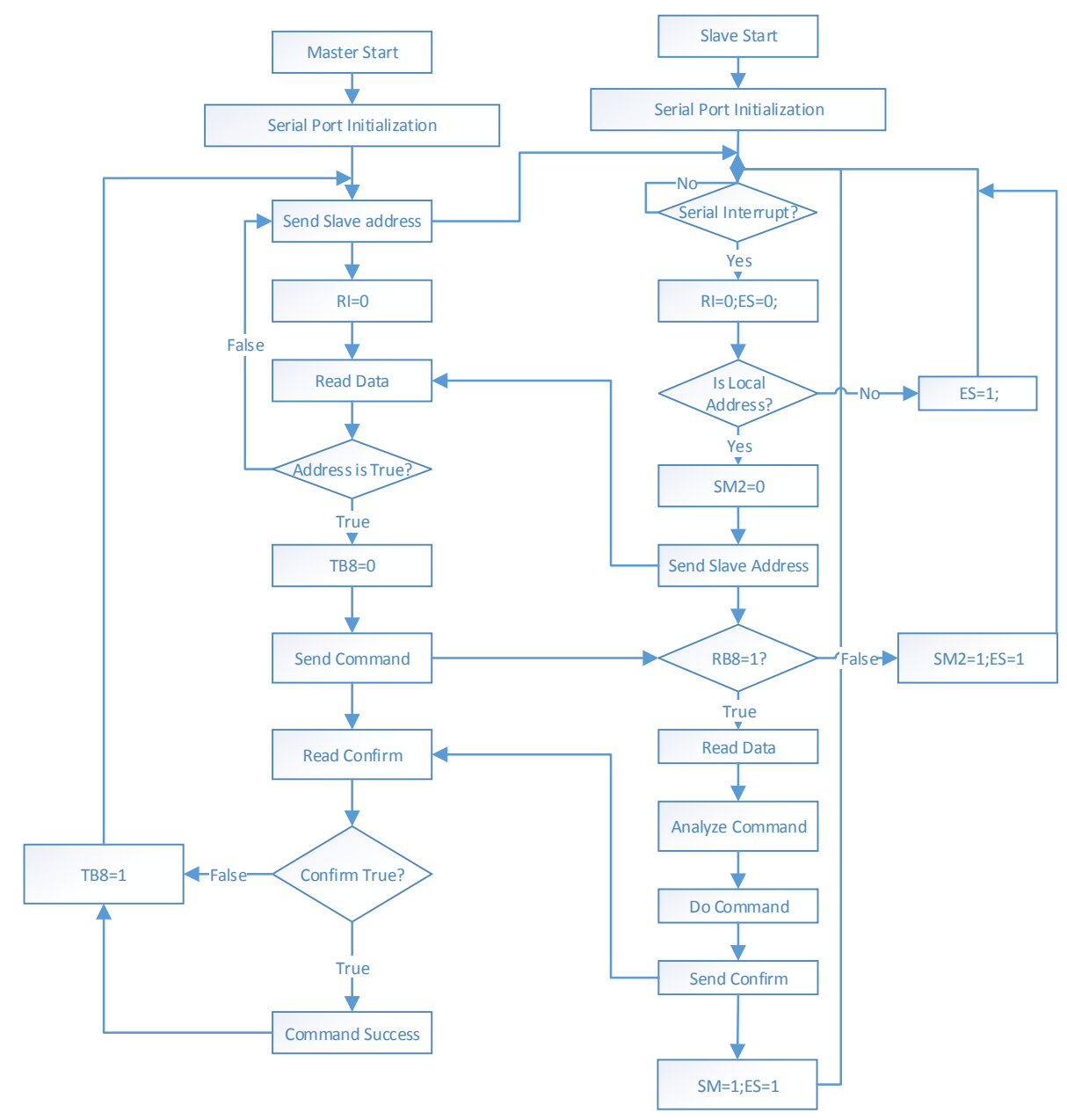

Figure 13. The Rs-485 Communication Work Flow

We by using the language C51 for programming the MCU to design the software of the controller. The software receive/send the communication data between the MCU and RS-485, processes the signals from various sensors, controls the movement of the mobile rack. The sample code of how to process the communication data and control command is shown next. 


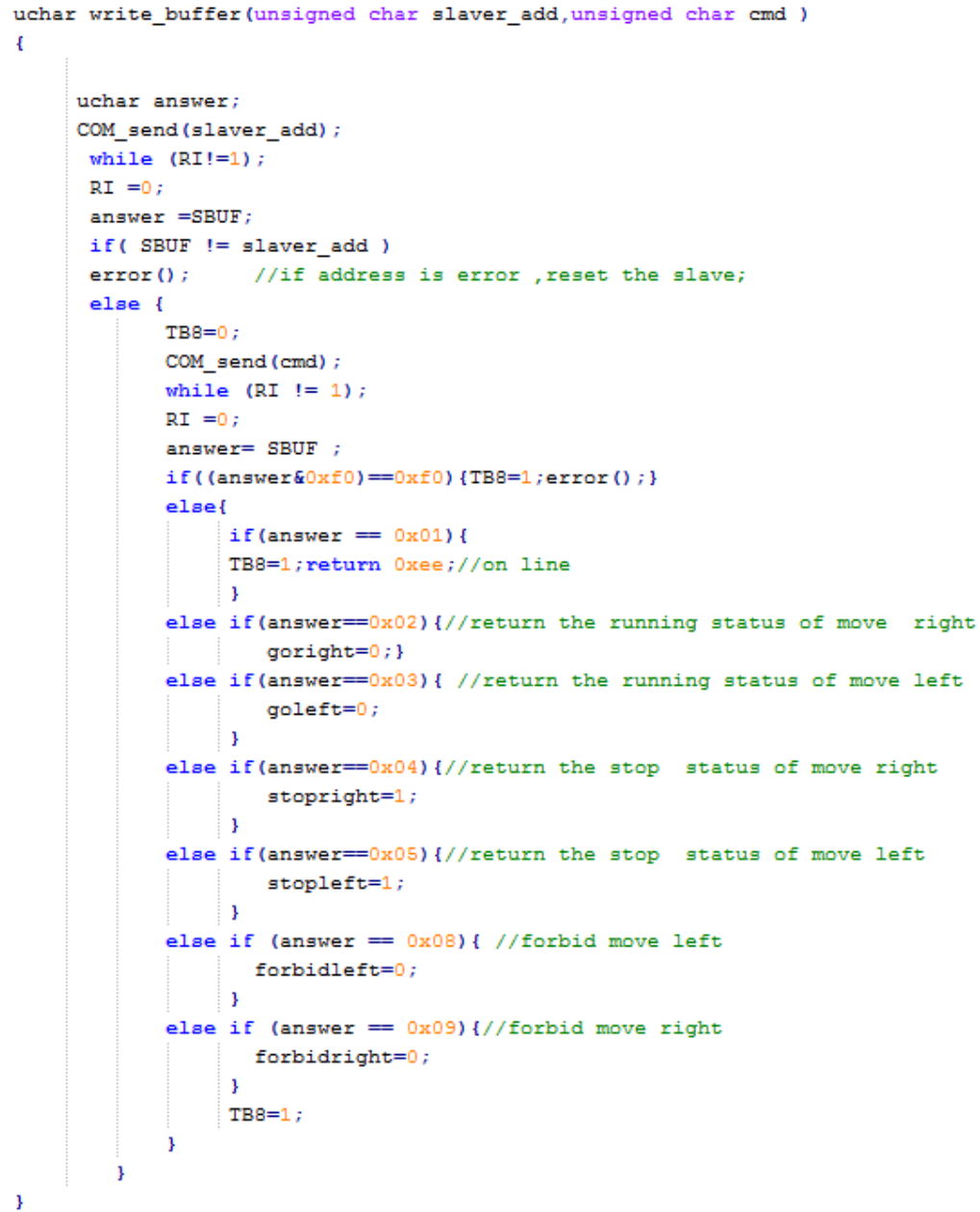

\subsection{Ultrasonic Ranging Algorithm Design}

The main title (on the first page) should begin $13 / 16$ inches (7 picas) from the top edge of the page, centered, and in Times New Roman 14-point, boldface type. Capitalize the first letter of nouns, pronouns, verbs, adjectives, and adverbs; do not capitalize articles, coordinate conjunctions, or prepositions (unless the title begins with such a word). Please initially capitalize only the first word in other titles, including section titles and first, second, and third-order headings (for example, "Titles and headings" - as in these guidelines). Leave two blank lines after the title.

The ultrasonic transmitter transmits the ultrasonic wave in one direction; the timer of the MCU starts timing at the same time. The ultrasonic wave transmits in the air, on the way over obstacles immediately reflected. When the ultrasonic receiver receives reflected ultrasonic waves, the timer of the MCU will stop timing. So the distance D is:

$$
D=\frac{1}{2} c t
$$

where, $\mathrm{c}$ is the speed of the ultrasonic wave and $t$ is the time.

$$
c=\sqrt{\frac{\gamma R T}{M}}=c_{0} \sqrt{1+\frac{T}{273 K}}
$$

The distance is: 


$$
D=\frac{1}{2} c_{0} t \sqrt{1+\frac{T}{273 K}}=165 \cdot 7 t \sqrt{1+\frac{T}{273 K}}
$$

The MCU calculates the distance by the ultrasonic ranging module between two racks in order to prevent collisions. Figure 13 shows the hardware of the control circuit of the client controller.

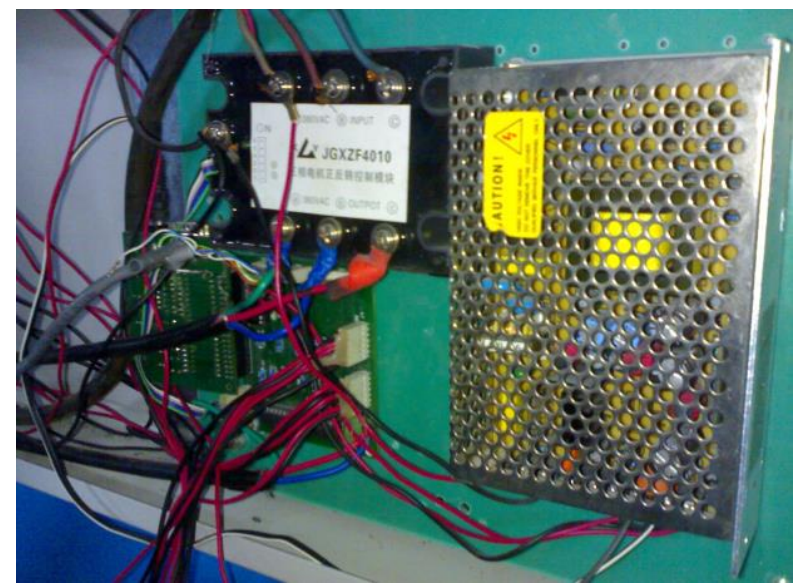

Figure 14. The Control Circuit of the Client Controller

This system has been running smoothly in a pharmaceutical company for 2 years. Figure 14 shows the scene of the application of the system
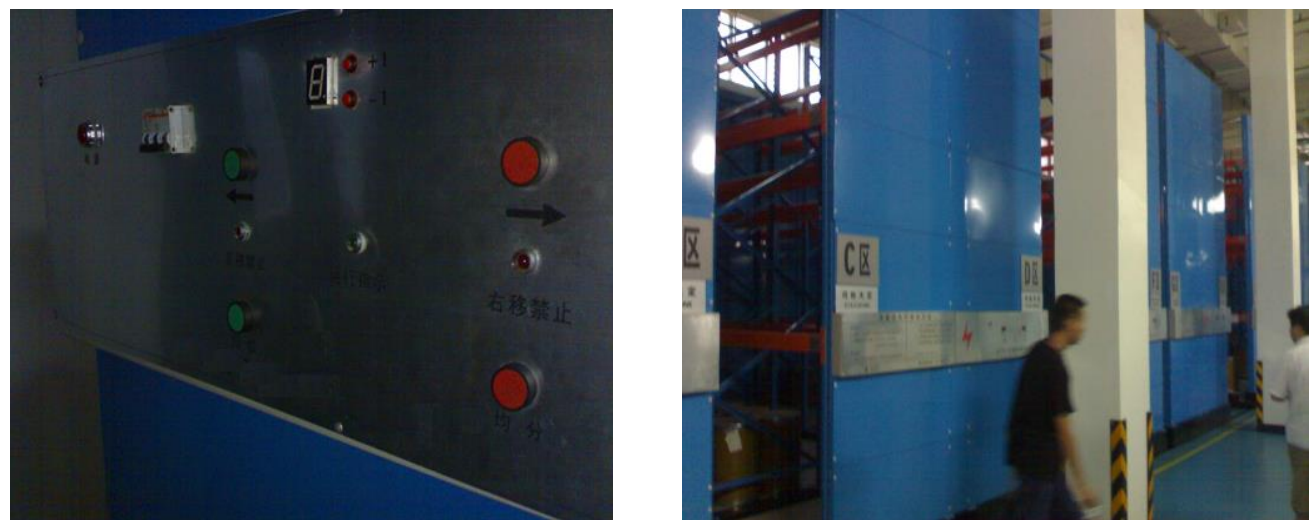

Figure 15. The Scene of the Application of the Mobile Rack

\section{Conclusion}

The new type electric-intensive mobile rack we designed can greatly reduce storage space and improve the efficiency of cargo access. In this paper, we design a controller based on the MCU, use the Rs-485 as the communication means, ultrasonic ranging device and photoelectric switch as the safety protection. The control software ensures the stability of the system. This system has been running smoothly in a pharmaceutical company for 2 years, proved the feasibility and robustness of the system.

\section{Acknowledgement}

We would like to acknowledge the National Natural Science Foundation of China (Grant No. 10902051, 11102089) and the Scientific Research Foundation of Nanjing University of Posts and Telecommunications (Grant No. NY213111). 


\section{References}

[1] Malmborg and J. Charles, "Rule of thumb heuristics for configuring storage racks in automated storage and retrieval systems design", International Journal of Production Research, vol. 39, no. 3, (2001), pp. 511-527.

[2] G. Marc, Mc G. Leon, S. Gunter, B. Doug and T. Lei, "Design models for warehousing systems with modular drawers and bin shelving systems", IIE Annual Conference and Exhibition, (2004); TX, United states.

[3] P. G. Wang, X. M. Qi, X. P. Zong, "Picking Route Optimization of Automated Warehouse Based on Improved Genetic Algorithms", Applied Mechanics and Materials, vol.4, no.11, (2013), pp.26942697.

[4] A. H. Guezzen, Z. Sari and L. Ghomri, "A study on Mobil racks automated storage and retrieval system (M-AS/RS)", Communications, Computing and Control Applications, International Conference on. IEEE, (2011).

[5] L. Yi-Zhong and H. Xue-Fei, "Appliance Research on New TypeMoving Control System of Horizontal Mobile Rack”, Mechanical \& ElectricalEngineering Magazine, vol.6, no.23, (2006), pp.4143.

[6] S. Sheel and O. Gupta, "High Performance Fuzzy Adaptive PID Speed Control of a Converter Driven DC Motor”, International Journal of Control and Automation, vol.5, no.1, (2012), pp.71-78. 